\title{
Investigation of Leish-111f Epitopes to Design a New Multi-epitope Vaccine Against Leishmania Major Using Immunoinformatics Approaches
}

\author{
Mahsa Rabienia \\ Fasa University of Medical Science \\ Rasoul Daneshi \\ Fasa University of Medical Science \\ Nahid Mortazavidehkordi \\ Fasa University of Medical Science \\ Ali Ghanbariasad \\ Fasa University of Medical Science \\ Abbas Abdollahi \\ Fasa University of Medical Science \\ Zahra Roudbari \\ University of Jiroft \\ Akbar Farjadfar ( $\square$ A.farjadfar@fums.ac.ir) \\ Fasa University of Medical Science
}

\section{Research Article \\ Keywords: multi-epitope vaccine, leishmaniasis, docking, molecular dynamics simulation}

Posted Date: January 15th, 2021

DOI: https://doi.org/10.21203/rs.3.rs-146687/v1

License: (1) This work is licensed under a Creative Commons Attribution 4.0 International License. Read Full License 


\section{Abstract}

\section{Background}

Since the incidence of various types of leishmaniasis, no definitive treatment has been considered for the disease, and due to its high prevalence worldwide, this issue has caused many concerns. Cutaneous leishmaniasis is the most common form of the disease which, can cause malignant lesions on the skin. Vaccination for the prevention and treatment of leishmaniasis can be the most effective way to combat this disease. In this study, we designed a new multi-epitope vaccine using immunoinformatics tools, which confirmed its effectiveness in the in silico.

Methods

Sequences Leish-111f protein (TSA, Leif, and LMSTI1) of Leishmania major (L. major) were downloaded from GenBank and with the help of immunoinformatic tools, was designed a new multi-epitope vaccine antigen of $L$. major.

Result

Th and Tc epitopes of the leish-111f protein were predicted using bioinformatics tools. The final multi epitope was consisted of $18 \mathrm{CTL}$ epitopes that joined by AAY linker. There are also $9 \mathrm{HTL}$ epitopes in the structure of the final vaccine that were joined by GPGPG linker. The profilin adjuvant was also added into the construct by AAY Linker. There were 613 residues in the structure of the final construct. The multi epitope was stable and non-allergic. the data obtained from the binding of final multi-epitope vaccine-TLR11 residues (band lengths and weighted scores) showed that the ligand and the receptor have a high affinity to bind to each other. Moreover, in silico cloning approach, was improved the expression of proposed vaccine in E. coli host. Codon adaptation index and GC percent were calculated 1.0 and 53.35, respectively

Conclusion

Based on these results, we hope that the multi-epitope vaccine, which contains the most appropriate epitopes of a strong Leishmania major immunogen, along with an adjuvant capable of binding to TLR11, will further stimulate the immune system against the L.major.

\section{Background}

The worldwide outbreak of leishmaniasis, which has endangered 98 countries and a population of about 350 million people over the years, has led to many efforts to solve this health crisis (1). Unfortunately, despite many studies about leishmaniasis, there is still no definitive treatment for this zoonotic disease in human $(2,3)$. Drugs such as pentavalent antimony $\left(\mathrm{Sb}^{5+}\right)$, pentamidine, amphotericin $\mathrm{B}$ and miltefosine are used to combat against types of the disease, but due to high cost and side effects, they cannot be prescribed with confidence $(4,5)$. In addition, many vaccines were successful in vitro, but their effectiveness has not been confirmed in later stages of research and few vaccines such as LEISH-111f and ChAd63KH DNA vaccine have entered into clinical trials $(6,7)$.

Understanding the host defense mechanism against the parasite is essential in the production of an effective vaccine. Studies on leishmaniasis have shown that stimulation of cellular immunity is essential for defense against the parasite(8). Once the parasite enters the host, macrophages M1 or M2 play a key role in the parasite's defense or survival. If the T helper 1 (Th1) response is activated, the cytokines IL-12, IL-2, IL-17, IFNY and TNF-a are increased. The presence of these cytokines, especially IFNy, leads to the differentiation of M1 macrophages, the production of NO by iNOS and finally the elimination of the parasite. On the other hand, by directing the immune response to T helper 2 (Th2), cytokines such as IL-4, IL-13, and IL-10 are produced, which inhibit IFN-y production and differentiate M2 macrophages. This route causes the infection to remain in the body due to the inefficiency of the immune system in parasitic defense ( 9 , 10). Also, in some studies, an increase in humoral immune response has been demonstrated by IgG2a antibody production to defense against leishmaniasis (11). Therefore, vaccination aimed at stimulating Th1, T cytotoxic (Tc), and humoral immune responses can be highly effective in defense against the parasite.

Several Leishmania antigens have been used to synthesize a variety of vaccines against the disease, and some of these vaccines have shown good immunogenicity in the in vitro. One of these antigens is Leish-111f containing $L$. major Thiol-specific Antioxidant (TSA), $L$. Major stress inducible 1 (LMSTI1), and L. brazilensis elongation initiation factor (Leif) (12). In five studies, immunogenicity evaluation was performed using a vaccine containing Leish-111f antigen with MPL-SE adjuvant and all of them in clinical trials were successful (13).

These immunogens can be used to make a variety of vaccines, including protein, DNA and mRNA vaccines. As mentioned, the Leish-111f protein has been shown to be effective and can stimulate the immune system well, so it is a good candidate for synthesizing other types of 
vaccines. On the other hand, due to the large size of the genome of this polyprotein, it is better to use selected epitopes of its proteins in the synthesis of the vaccine, so that if it becomes a candidate for the synthesis of the DNA vaccine against Leishmania, it can easily enter the AAV (adeno-associated virus) vectors (14).

Nowadays, with the advancement of immunoinformatics approach, vaccines can be designed in the in silico that their effectiveness is confirmed before being tested in the in vitro and in vivo. According to the prediction methods of immunoinformatics, the most suitable epitopes of each immunogens are selected for vaccine design. Thus, it can decrease the cost of vaccine synthesis along with increase of safety, specify, and effectiveness of vaccines (15). Also, using a multi-epitope instead of a complete protein vaccine can reduce allergic reactions and is a good replacement to a protein vaccine. Moreover, the infectious agents present in the synthesis of attenuated live vaccines are not present in the synthesis of these vaccines (16). Therefore, we can design and synthesis a new multi-epitope vaccines against the disease using this therapeutic tool (17).

In this study, for the first time, we designed a multi-epitope vaccine using Leish- $111 \mathrm{f}$ immunogenic antigen of $L$. major to combat against cutaneous leishmaniasis with the help of immunoinformatic tools. To increase the immunogenicity of the vaccine, we also used a profilin adjuvant that is a TLR11-agonist and can be attached at the level of macrophages and monocytes. For this purpose, the most suitable HTL and CTL epitopes of Leish-111f, were predicted and linked them by AYY and GPGPG linkers. Also, profilin with the AAY linker was added to the selected epitopes. The designed vaccine was tested in several steps containing B-cell epitope prediction, tertiary structure prediction, tertiary structure validation and refinement, molecular docking of final vaccine and TLR11, molecular dynamics simulation and codon optimization.

\section{Methods}

\section{Study design}

To design a new multi-epitope vaccine, several steps were performed: Prediction of HTL and CTL epitopes; Binding the selected T cell epitopes by using suitable linkers, and designing of final vaccine construct; B cell epitope prediction; docking analysis between the final vaccine and TLR11; molecular dynamics simulation. Finally, in silico cloning in E. coli was performed. The schematic diagram of the designed vaccine has been shown in Fig. 1.

\section{Protein sequence of Leish-111f polyprotein}

The amino acid sequences of leish-111f polyprotein containing TSA, LmSTI1, and Leif proteins with accession numbers (AF044679, MH061281, and W5XL77) and also profilin (PDB ID: AAX33672.1) as an adjuvant were obtained from GenBank, protein database of the National Center for Biotechnology Information (NCBI, http://www.ncbi.nih.gov/genbank/).

\section{T-cell epitope prediction}

To predict the CTL and HTL epitopes of TSA, LMSTI1, and Leif proteins, the IEDB server was used. Prediction of 9-mer and 15-mer epitopes to bind to $\mathrm{MHCl}$ and $\mathrm{MHCll}$ were done by using IEDB (http://tools.iedb.org/mhcii/). The server predicts the CTL and HTL epitopes on the basis of SMM (stabilized matrix method), Artificial Neural Networks (ANN) and, Allele specific affinity cutoff (IC50)(18). In this study, we selected the mouse alleles to bind the $\mathrm{MHCl}$ and $\mathrm{MHCl}$.

\section{IFN-y inducing epitope prediction}

Using IFN epitope server (http://crdd.osdd.net/raghava/ifnepitope/), prediction of interferon-gamma inducing epitopes of HTL epitopes was performed. The server predicts the epitopes based on the methods such as SVM based, motif based, and hybrid approach. On this server, IFN-y inducing and non-inducing MHC-II binders were determined.

\section{Construction of final multi-epitope vaccine}

Using the in silico information, the final multi-epitope vaccine was designed. First, AYY and GPGPS flexible linkers were used to joined HTL and CTL epitopes, respectively (19). Then, to enhance the immunogenicity of final multi-epitope vaccine, profilin was added as an adjuvant to the $\mathrm{N}$-terminal of the construct by using AYY linker.

\section{Conformational and Linear B cell epitope prediction}


According to the 3-D structure of the construct, the ElliPro suite (http://tools.iedb.org/ellipro/) was used to predict of conformational and linear B cell epitopes of the final vaccine construct (20).

The server assigns a score to each predicted epitope, named PI (Protrusion index). First, the three-dimensional shape of the protein is estimated by a number of spherical ellipses, which the higher the percentage, the fewer residues outside the ellipsoid. The PI value is defined based on the center of mass remaining outside the largest possible ellipsoid and residues with higher scores have more access to the solvent. Discontinuous epitopes are characterized by PI values and clustered by R distance (in $\AA$ between remaining mass centers). The higher the $\mathrm{R}$ value, the larger the epitopes.

\section{Tertiary structure prediction of final multi-epitope vaccine}

I-TASSER Server (Iterative Threading ASSEmbly Refinement) available at (http://zhanglab.ccmb.med.umich.edu/ I-TASSER/) was used to predict of tertiary structure of the final vaccine construct. I-TASSER predicts the best model with the highest C-score for further analysis. This server provides a PDB file that uses the multiple threading approach LMOTS and predicts the 3D structure using iterative templatebased pattern assembly simulations of full-length atomic models. Moreover, the server was the best in terms of function prediction in CASP9 (21).

\section{Tertiary structure refinement of final multi-epitope vaccine}

To refine of 3D model of the final vaccine construct, GalaxyRefine server available at (http://galaxy.seoklab.org/) was applied (22). Mild and aggressive relaxation methods are used to reconstruct the side chain residues, repackaging and molecular dynamics simulation of proteins. The server can also enhance the local and global quality of the produced models by I-TASSER (22).

\section{Validation of tertiary structure of final multi-epitope vaccine}

PROCHECK (http://servicesn.mbi.ucla.edu/PROCHECK/), ProSA (http://prosa.services.came.sbg.ac.at/prosa.php), and ERRAT (http://services.mbi.ucla.edu/ERRAT) servers were used to validate the 3D structure of the final vaccine construct. The PROCHECK gave a Ramachandran plot and assessed the stereochemical quality of the refined model (23). Furthermore, the ProSA provided an overall quality score based on Z-score value. It should be noted that scores outside the range of native proteins are not acceptable (24).

\section{Evaluation of physico-chemical properties of the final vaccine construct}

ProtParam (http://web.expasy.org/protparam/), an online web server was used to calculate the physicochemical characteristics of final multi-epitope vaccine containing aliphatic index, instability index, theoretical PI (isoelectric point) molecular weight, in vitro and in vivo halflife, and grand average of hydropathicity (GRAVY)(25). Then, the solubility of the final vaccine construct over expression in E. coli was calculated by using SOLpro (http://scratch.proteomics.ics.uci.edu/)(26).

\section{Allergenicity and antigenicity assessment of the final vaccine construct}

AllerTOP v2.0 and AllergenFP1.0 were applied to determine the antigenicity of the final vaccine construct. Using five E-descriptors, including amino acid hydrophobicity, size, amino acid tendency to helix, amino acid abundance, and tendency of $\beta$-strand formation, the amino acids in the protein sequence are described in the data set of AllergenFP1.0 server. Also, based on auto-cross covariance (ACC) transformation, the strings were transformed into uniform vectors. The server categories the proteins in two allergen and non-allergen, according to Tanimoto coefficient (27). AllerTOP v2.0 also used the same five E-descriptors that mention above. This server classifies the protein to allergen and non-allergen based on k-nearest neighbor algorithm (kNN, k=1)(28). Thereafter, the determination of antigenicity was done by using ANTIGENpro (http://scratch.proteomics.ics.uci.edu/) and VaxiJen v.2 (http://www.ddgpharmfac.net/) servers. ANTIGENpro is an alignment-free and sequence-based server. The accuracy of this pathogen independent server is $82 \%(29)$. Protective antigens were also predicted by VaxiJen, a first alignment-independent prediction(30) .

\section{Docking analysis of final vaccine and TLR-11}

ClusPro server available at (http://cluspro.bu.edu/login.php) was applied to determine the affinity of binding between ligand (final multiepitope vaccine) and receptor (TLR-11). Elimination of unstructured protein regions, use of attraction or repulsion, calculation of pairwise distance restraints, and homo-multimers construction, are some of the parameters that this server considers to provide docking results of two proteins. In addition, the selection of highly populated cluster centers of low-energy structures is another feature of this server (31).

Finally, visualization of docked complex was performed by PyMOL program. 
The structural properties and interaction of ligand (the final vaccine construct) and receptor (TLR-11) was evaluated in the in vivo conditions through molecular dynamics simulation. In this step, Using GROMOS 54a7 and 43a1 package, docked complex of final vaccine and TLR-11 was simulated during 50000 picoseconds (Ps) at $310^{\circ} \mathrm{K}$ and 1 bar pressure. A Cubic box with periodic boundary conditions with water molecules of SPC/E was also used. Moreover, to minimize of system energy and relaxation of solvent molecule the steepestdescent algorithm was applied. Also, fixation of the atom was performed by using A LINear Constraint Solver (LINCS) algorithm and SETTLE, an analytical algorithm, was used for solvent molecules. To calculate of total electrostatic energy and other non-bonded interactions, Particle Mesh Ewald (PME) summation method and L-J model (with $10 \mathrm{~A}^{\circ}$ cutoff distances), were used, respectively. Moreover, the temperature and pressure of each system, at the time of coupling equal to $0.1 \mathrm{Ps}$

were preserved using Berendsen weak coupling algorithm. Finally, the root mean square deviation (RMSD) and root mean square fluctuation (FMSF) diagrams were plotted and the fluctuations and stability of the docked complex were investigated.

\section{In silico cloning of final multi-epitope vaccine}

For this purpose, SnapGene tool was applied and CDNA was provided as an output of the server. The cDNA sequence was used to determine of CAI value and GC content. The CAI value higher than 0.8 and GC content between $30-70 \%$ are acceptable. Then, $X$ hol and $N d e l$ restriction enzymes were added to the CDNA sequence. Finally, codon optimization in to pET28a (+) vector was done.

\section{Results}

\section{T-cell epitope prediction}

Using IEDB server, CTL epitopes of leish111-f consisting three proteins was predicted. Six epitopes were predicted from each protein of leish111f. Therefore, a total of 18 epitopes with the lowest percentile rank was selected. Also, a total of $9 \mathrm{HTL}$ epitopes of all proteins was selected that for each protein three epitopes were selected (Table 1, 2). Moreover, with the help of IFNepitope server, 12 HTL epitopes was determined to induce of IFN- $\boldsymbol{y}$. (Table 3)

Table 1. Predicted CTL epitope with the lowest percentile rank

\begin{tabular}{|c|c|c|c|c|c|}
\hline Protein & alleles & Start & End & Sequence & Percentile rank \\
\hline \multirow{6}{*}{ TSA } & $\mathrm{H} 2-\mathrm{Db}$ & 48 & 56 & FSFVCPTEV & 1.00 \\
\hline & $\mathrm{H} 2-\mathrm{Dd}$ & 51 & 59 & VCPTEVIAF & 0.30 \\
\hline & $\mathrm{H} 2-\mathrm{Kb}$ & 38 & 46 & WVVLFFYPL & 0.17 \\
\hline & $\mathrm{H} 2-\mathrm{Kd}$ & 81 & 89 & EYAHLQWTL & 1.2 \\
\hline & $\mathrm{H} 2-\mathrm{Kk}$ & 145 & 153 & NDMPVGRSV & 2.00 \\
\hline & $\mathrm{H} 2-\mathrm{Ld}$ & 21 & 29 & MPNGSFKKI & 1.25 \\
\hline \multirow{6}{*}{ ImST1 } & $\mathrm{H} 2-\mathrm{Db}$ & 395 & 403 & YSNRAAAYI & 0.20 \\
\hline & $\mathrm{H} 2-\mathrm{Dd}$ & 252 & 260 & KDPNNTLYI & 2.00 \\
\hline & $\mathrm{H} 2-\mathrm{Kb}$ & 400 & 408 & AAYIKLGAF & 0.35 \\
\hline & $\mathrm{H} 2-\mathrm{Kd}$ & 258 & 266 & LYILNVSAV & 0.4 \\
\hline & $\mathrm{H} 2-\mathrm{Kk}$ & 349 & 357 & EEAYIDPEI & 0.27 \\
\hline & $\mathrm{H} 2-\mathrm{Ld}$ & 253 & 261 & DPNNTLYIL & 0.7 \\
\hline \multirow{6}{*}{ Leif } & $\mathrm{H} 2-\mathrm{Db}$ & 106 & 114 & LALQTAEVI & 0.33 \\
\hline & $\mathrm{H} 2-\mathrm{Dd}$ & 164 & 172 & RGALRTESL & 0.20 \\
\hline & $\mathrm{H} 2-\mathrm{Kb}$ & 191 & 199 & QIYEIFRFL & 0.42 \\
\hline & $\mathrm{H} 2-\mathrm{Kd}$ & 385 & 393 & HYHTQIDEL & 1.10 \\
\hline & $\mathrm{H} 2-\mathrm{Kk}$ & 239 & 247 & LEGIKQFFI & 0.12 \\
\hline & H2-Ld & 23 & 31 & RPIPSFDDM & 0.16 \\
\hline
\end{tabular}

Page 5/15 
Table 2. Predicted HTL epitopes with the lowest percentile rank

\begin{tabular}{|c|c|c|c|c|c|}
\hline Protein & alleles & Start & End & Sequence & Percentile \\
\hline \multirow{3}{*}{ TSA } & H2-IAd & 95 & 109 & GGLGTMAIPMLADKT & 0.23 \\
\hline & $\mathrm{H} 2-\mathrm{IAb}$ & 44 & 58 & YPLDFSFVCPTEVIA & 4.36 \\
\hline & H2-IEd & 82 & 96 & YAHLQWTLQDRKKGG & 14.70 \\
\hline \multirow{3}{*}{ ImST1 } & H2-IAd & 310 & 324 & RQRKYEAAIDLYKRA & 0.28 \\
\hline & $\mathrm{H} 2-\mathrm{IAb}$ & 368 & 382 & YFKEDKFPEAVAAYT & 9.46 \\
\hline & H2-IEd & 68 & 82 & PNWAKGYVRRGAALH & 14.21 \\
\hline \multirow{3}{*}{ Leif } & H2-IAd & 103 & 117 & TRELALQTAEVISRI & 0.28 \\
\hline & $\mathrm{H} 2-\mathrm{IAb}$ & 39 & 53 & RGIYSYGFEKPSSIQ & 2.18 \\
\hline & H2-IEd & 267 & 281 & IAQSVIFANTRRKVD & 14.48 \\
\hline
\end{tabular}

Table 3. Predicted of IFN-gamma inducing epitopes

\begin{tabular}{|lllll|}
\hline No & Epitope & Start & End & Score \\
\hline 1 & CPTEVAAYVCPTEVI & 4 & 19 & 0.68 \\
\hline 2 & AYEYAHLQWTLAAYN & 34 & 49 & 0.34 \\
\hline 3 & AYMPNGSFKKIAAYY & 58 & 73 & 0.05 \\
\hline 4 & AAYYSNRAAAYIAAY & 69 & 84 & 0.75 \\
\hline 6 & AYAAYIKLGAFAAYL & 94 & 109 & 0.52 \\
\hline 7 & PIKLGAFAAYLYILN & 98 & 113 & 0.13 \\
\hline 9 & AHLQGGGLTMAIPMLA & 214 & 229 & 0.29 \\
\hline 10 & PGYFKEDKFPEAVAA & 295 & 310 & 0.09 \\
\hline 11 & NWAKGYVRRGAALHG & 318 & 333 & 0.03 \\
\hline 12 & EKPSIQGPGPGIAQS & 365 & 380 & 0.55 \\
\hline
\end{tabular}

\section{Construction of final multi-epitope vaccine}

A total of 9 CTL epitopes was linked together by AAY linker. Also, GPGPG linker was utilized to join the HTL epitopes with them. Then, profilin with the length of 163 amino acid was added at the $\mathrm{N}$ terminal of the construct. The construct was shown in Fig. 1.

\section{B cell epitope prediction of final multi-epitope vaccine}

Prediction of linear and discontinuous epitopes of the final vaccine construct was performed using ElliPro suite. According to the results obtained from the server, 12 linear and 7 discontinuous B cell epitopes were predicted in final vaccine that can stimulate humoral immunity (Fig. 2 and table 4, 5).

Table 4. Predicted of linear B cell epitopes 


\begin{tabular}{|c|c|c|c|c|c|}
\hline No & Start & End & Peptide & Number & Score \\
\hline 1 & 536 & 556 & QGPGPGIAQSVIFANTRRKVD & 21 & 0.811 \\
\hline 2 & 35 & 72 & AADDDDGWSKLYKDDHEEDTIGEDGNACGKVSINEAST & 38 & 0.777 \\
\hline 3 & 134 & 153 & ALYDEEKEQDKGNSRTSALA & 20 & 0.76 \\
\hline 4 & 327 & 395 & SLAAYQIYEIFRFLAAYHYHTQIDELAAYLEGIKQFFIAAYRPIPSFDDMGPGPGGGLGTMAIPMLADK & 69 & 0.761 \\
\hline 5 & 419 & 437 & GPGYAHLQWTLQDRKKGGG & 19 & 0.719 \\
\hline 6 & 9 & 20 & KEWLVDTGYCCA & 12 & 0.717 \\
\hline 7 & 75 & 122 & AAVDDGSAPNGVWIGGQKYKVVRPEKGFEYNDCTFDITMCARSKGGAH & 48 & 0.704 \\
\hline 8 & 449 & 455 & AIDLYKR & 7 & 0.678 \\
\hline 9 & 516 & 530 & IGPGPGRGIYSYGFE & 15 & 0.675 \\
\hline 10 & 403 & 410 & PLDFSFVC & 8 & 0.64 \\
\hline 11 & 462 & 471 & YFKEDKFPEA & 10 & 0.613 \\
\hline 12 & 491 & 499 & RGAALHGPG & 9 & 0.599 \\
\hline
\end{tabular}

Table 5. Predicted conformational B cell epitopes

\begin{tabular}{|c|c|c|c|}
\hline No & Residues & $\begin{array}{l}\text { Number } \\
\text { of } \\
\text { residues }\end{array}$ & Score \\
\hline 1 & _:F548,_:A549, _:N550,_:T551, _:R552,_:K554,_:V555, _:D556 & 8 & 0.948 \\
\hline \multirow[t]{2}{*}{2} & 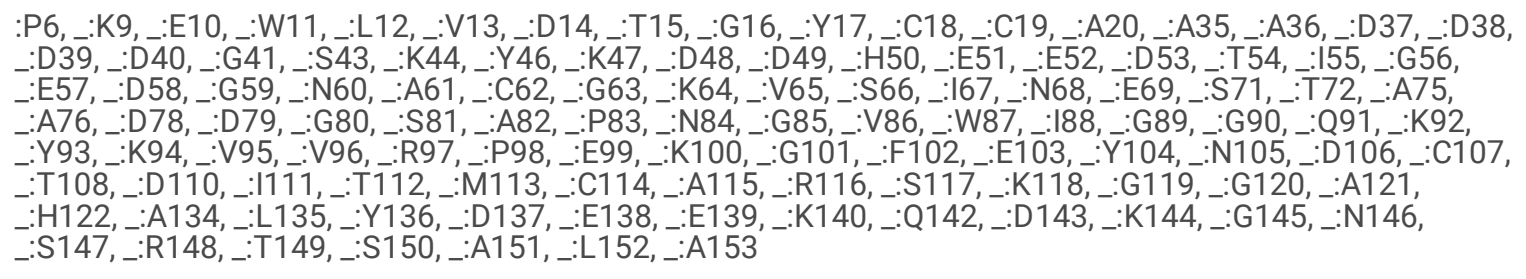 & & \\
\hline & & 113 & 0.746 \\
\hline \multirow[t]{2}{*}{3} & :Q536,_:G537,_:P538, _:G539, _:P540,_:G541,_:I542,_:A543,_:Q544, _:S545, _:V546, _:I547 & 12 & 0.705 \\
\hline & 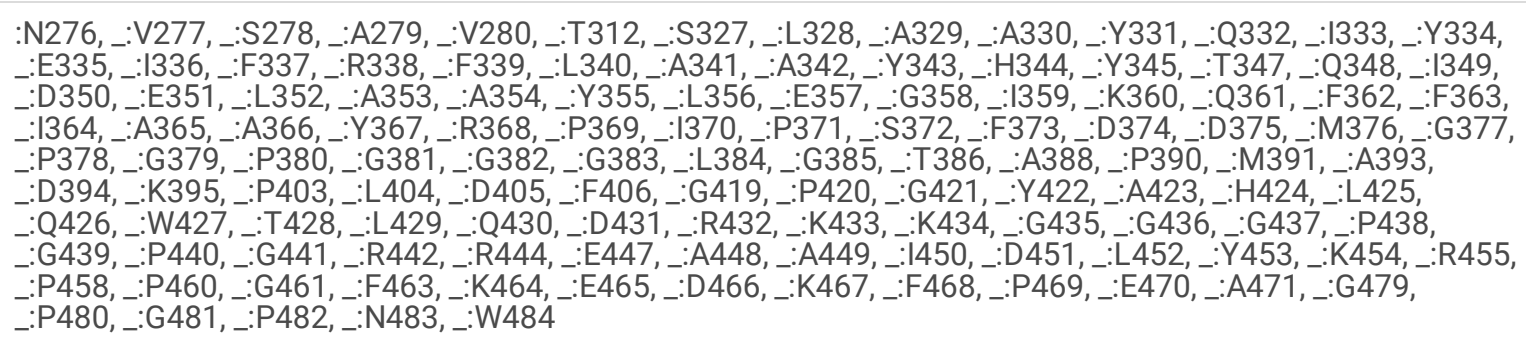 & & 0.688 \\
\hline 4 & & 127 & \\
\hline 5 & $\begin{array}{l}: I 516, \text { _:G517, _:P518, _:G519, _:P520,_:G521, _:R522,_:G523,_:I524,_:Y525, _:S526, _:Y527,_:G528, _:F529, } \\
\text { _:E530 }\end{array}$ & 15 & 0.675 \\
\hline 6 & :S407,_:F408,_:V409,_:C410 & 4 & 0.625 \\
\hline 7 & :R491, _:G492, _:A493, _:A494,_:L495, _:H496, _:G497,_:P498,_:G499 & 9 & 0.599 \\
\hline
\end{tabular}


I-TASSER results showed five predicted models for the 3D structure of the vaccine (3necA, 3tkpA, 3nec, 6tdaL, 3nec). Among them, the model 1(PDB Hit: 3nec) was recognized as the best model with the C-score of -2.23 (Fig. 3A). Then, the tertiary structure of the predicted model of the final vaccine construct, was also refined by using Galaxy Refine server and the scores of model quality was calculated. Based on the GDT-HA ( 0.9545 ), RMSD ( 0.435 ), MolProbity (1.825), Clash score (13.1), Poor rotamers (0.7) and Rama favored (96.0),, model 1 was selected as the best model for the final vaccine construct. Then, Ramachandran plot, ProSA and ERRAT tools were used to validate of the selected refine model. For this purpose, verification of quality and potential errors of the crude 3D model was done. ERRAT tool showed the value of $86.42 \%$ as an overall quality factor of refined model. The ProSA was calculated a Z-score of -4.55 , that is a plausible value of native proteins as shown in Fig. 3B. In addition, the data obtained from the Ramachandran diagram showed $87 \%$ residues located in most favored regions, $9.9 \%$ residues in additional allowed regions, and $1.5 \%$ residues in allowed and $1.5 \%$ residues in disallowed regions as shown in Fig. $3 \mathrm{C}$.

\section{Assessment of physico-chemical characteristics and solubility of the final vaccine construct}

ProtParam tool was used to determine the physicochemical properties of the final vaccine construct. The values of theoretical Isoelectric point $(\mathrm{PI})$, and molecular weight were estimated 5.17 and $60.08 \mathrm{KDa}$, respectively. The amount of PI revealed the final vaccine construct is acidic in nature. The total number of negatively and positively charge was 61 and 47, respectively. Another data in this step such as half-life in mammalian reticulocytes were 30 hours in mammalian reticulocytes, in vitro, $>20$ hours in yeast and $>10$ hours in E. coli, in vivo. Also, instability index and aliphatic index were computed 29.68 and 76.17, respectively. The stability and thermostability of the construct in nature was confirmed by the values of attained from instability index and aliphatic index. The amount of Grand average of hydropathicity (GRAVY) that was -0.157 showed the construct is hydrophilic in nature. Then, SOLpro server was utilized to investigate of the final vaccine construct solubility. The solubility of vaccine in E. coli was confirmed by the value of 0.90 .

\section{Evaluation of allergenicity and antigenicity of final multi-epitope vaccine}

Allergenicity assessment was done using AllergenFP1.0 and AllerTOP servers. The results showed this the final vaccine construct was nonallergen. Moreover, antigenicity of the final construct was carried out by VaxiJen v.2 and ANTIGENpro servers. Based on the result of VaxiJen at 0.4 threshold in virus model and ANTIGENpro, the probability of the final vaccine was confirmed by the value of 0.42 and 0.65 respectively.

\section{Docking study between final multi-epitope vaccine and TLR-11}

ClusPro v. 2.0 server was used to molecular docking of final multi-epitope vaccine with the TLR11. Based on biophysical properties of ligand (multi-epitope vaccine) and receptor (TLR11) a total of 30 models were predicted. But among all of the predicted models the model number 0.00 with the lowest energy score was selected. The weighted score obtained was determined $-1990.8 \mathrm{kcal}$ that has the highest binding affinity in all of the predicted models. In final vaccine-TLR11 docked complex, Leu 158 (0) from final multi-epitope vaccine and Arg $767(\mathrm{HH} 12)$ from TLR11 revealed a hydrogen bond with length of $2.1 \AA$. Also, Tyr $163(\mathrm{H})$ from final vaccine and Asp $775(\mathrm{OD} 1)$ from TLR11 formed a hydrogen bands with the length of $1.9 \AA$. Other interactions containing Tyr 163 (O) - Arg 833 (HH21, HE) revealed two hydrogen bands at $2.0 \AA$ and $1.9 \AA$, respectively. Tyr $163(\mathrm{HH})$ - Ser $919(\mathrm{OH})$ indicated a hydrogen bands at $2.0 \AA$. Tyr $169(\mathrm{OG}, \mathrm{HH})$ from final vaccine were also indicated two hydrogen bands at $2.7 \AA$ with Gly $922(\mathrm{HH} 1)$ and Gly 924 (OH) from TLR11, respectively. (Fig. 4, Table $6)$.

Table 6. Interactions of docking analysis between final vaccine and TLR11 


\begin{tabular}{|c|c|c|}
\hline Bond length $(\AA)$ & Vaccine Construct- TLR11 atom pair & Lowest energy(kcal) \\
\hline $2.1 \mathrm{~A}$ & Leu (158) O: Arg (767) HH12 & \\
\hline \multirow[t]{2}{*}{$1.9 \mathrm{~A}$} & Tyr (163) H: ASP (775) OD1 & \\
\hline & & -1990.8 \\
\hline $2.0 \mathrm{~A}$ & Try (163) O: Arg (833) HH21 & \\
\hline $1.9 \mathrm{~A}$ & Try (163) O: Arg (833) HE & \\
\hline $2.0 \mathrm{~A}$ & Tyr (163) HH: Ser (919) OH & \\
\hline $2.7 \mathrm{~A}$ & Tyr (169) OG: GLY (922) HH1 & \\
\hline $2.7 \mathrm{~A}$ & Tyr (169) HH: GLY (924) OH & \\
\hline
\end{tabular}

\section{Molecular dynamics simulation of final multi-epitope vaccine}

The stability of the docked complex of the final multi-epitope vaccine and TLR-11 was assessed in vivo conditions by using molecular dynamics simulation methods. The fluctuation of the RMSD plot of the docked complex started about $0.2 \mathrm{~nm}$ and after a series of fluctuations, the stability of the docked complex was observed in 20000 picosecond (ps) and it was stable until the end of 50000 ps at 0.27 $\mathrm{nm}$. The RMSF plot showed fluctuations started at about $0.18 \mathrm{~nm}$ and after mild variation with an average of $0.15 \mathrm{~nm}$ ended at $0.2 \mathrm{~nm}$. The peak of the graph was also observed at $0.45 \mathrm{~nm}$. The plot was also obtained during 50000 ps (Fig. 5).

\section{In silico codon optimization of final multi-epitope vaccine}

Based on the results of the Java Codon Adaptation tool (JCat), reverse translation and codon optimization in E.coli (stain K12) was carried out. After the design of CDNA, codon adaptation index and GC percent were calculated 1.0 and 53.35 , respectively. The value of GC content was in acceptable rang of the results that is $30-70 \%$. Also the CAl value showed the vaccine is capable of the highest expression in the pET28a (+) vector (Fig. 6).

\section{Discussion}

There is no definitive treatment for leishmaniasis, and the cutaneous form of the disease, which affects 1.5 - 2 million people annually, can cause several lesions on the patient's skin if it progresses $(32,33)$. Researchers are still trying to make a vaccine for prevention and treatment of the disease(34). Identifying the host defense pathways against the pathogen and then strengthening these pathways can be the most important strategy in making an effective vaccine. To achieve this goal, immunoinformatics tools can be used to select the most appropriate epitope of the desired immunogens in less time with high accuracy (35), (36).

Leish-111f is an immunogen which a protein-based vaccine has already been approved and can be used as a candidate for another type of vaccine, such as a multi-epitope vaccine, which this type of vaccine is currently being considered in the treatment of various diseases including SARS-COV-2 (37).

The aim of this study was to design a new multi-epitope vaccine to be effective in combating leishmaniasis. In the first step, HTL and CTL epitopes of Leish111-f polyprotein from L. major were predicted and ligated using AYY and GPGPG linkers. Also, due to the fact that in the vaccination against leishmaniasis, the immune system needs to be more activated, we used the profilin as an adjuvant. The Profilin can 
bind to TLR11 at the macrophage and dendritic surface, and leading to innate immune stimulation, increased IFN- $\gamma$, and consequently increased Th1. Therefore, profilin can help increase the immunogenicity of this vaccine against Leishmania, which was our main goal.(38). There were also 12 epitopes in the final vaccine construct to induce interferon-gamma, which due to the role of interferon-gamma in inducing the immune system response against Leishmania, these epitopes can have a great effect on the defense against the parasite.

After the design of the final vaccine construct, conformational and linear B cell epitopes were predicted. The number of twenty linear and conformational epitopes in the structure of the final construct showed that this construct can stimulate the humoral immunity of the host well. Moreover, tertiary structure prediction of final vaccine construct along with validation and refinement was done. Based on the results of Ramachandran diagram and ProSA, the final vaccine construct was stable in nature. Also the quality and potential errors were predicted using a refinement of final vaccine.

Thereafter, on the basis of physicochemical properties of final vaccine, the values of PI and GRAVY were 5.17 and -0.157 , respectively that shows the construct is acidic and hydrophilic in nature. Also, thermostability of final vaccine was confirmed with the values of 5 and 10 for aliphatic index and instability index, respectively. Then, the solubility of the final multi-epitope vaccine in $E$. coli was done.

In addition, the data obtained from the binding of final multi-epitope vaccine-TLR11 residues (band lengths and weighted scores) showed that the ligand and the receptor have a high affinity to bind to each other. Also, the graphs of RMSD and RMSF indicated the docked complex was stable and has little variation. Therefore, the final multi-epitope vaccine can have a stable binding to TLR11 in the body through profilin.

In many vaccine design studies, docking and dynamic tests are performed to ensure the effectiveness of the construct. Because by confirming the affinity of binding between ligand-receptor by docking analysis in a vacuum condition and also ensuring the stability of this interaction in conditions similar to the body environment, the effectiveness of the vaccine in the in silico space is confirmed. Finally, the results of codon optimization (CAl value and GC content) showed that the multi-epitope vaccine could have the highest expression in the host.

\section{Conclusion}

In this study, immunoinformatic tools helped us design a new multi-epitope vaccine of Leish-111f of $L$. major. After identifying the most suitable epitopes of cellular immune stimulation of the host and adding an adjuvant to bind to TLR11 to increase the safety, the construct was designed. The B cell results showed that there are epitopes of humoral immune stimulation in the final construct and since humoral immunity can also be effective in defense against leishmaniasis, this vaccine can be a good candidate. Other tests confirmed the effectiveness of the vaccine. Moreover, docking analysis of final multi-epitope vaccine and TLR11 showed good interactions between them and molecular dynamics simulation of multi-epitope vaccine-TLR11 was also confirmed the stability of the docked complexes. Based on these results, we hope that the multi-epitope vaccine, which contains the most appropriate epitopes of a strong Leishmania major immunogen, along with an adjuvant capable of binding to TLR11, will further stimulate the immune system against the parasite. However, due to the effectiveness of the vaccine in the in silico, it is necessary to confirm the effectiveness of this vaccine in the later stages of the in vitro and in vivo.

\section{Abbreviations}

L.major: Leishmania major; 3D: Three dimensional; CAl: Codon adaptation index; CTL: Cytotoxic T lymphocytes; HTL: Helper T lymphocyte; TSA: Thiol-specific Antioxidant; leif: and L. brazilensis elongation initiation factor; LMSTI1: L. Major stress inducible 1 ; HLA: Human leukocyte antigen; MHC: Major histocompatibility complex; PDB: Protein Data Bank; pl: Isoelectric point; TLR11: Toll-like receptors-11

\section{Declarations}

\section{Acknowledgements}

The authors appreciate Fasa University of medical sciences for financial supports of this work. (Grant No. 97335)

\section{Authors' contributions}

A.F, Z.R and N.M conceived and designed this study; M.R, Z R and R.D performed the experiments; A.A, A.Gh, Z.R and N.M analyse the results; M.R wrote the manuscript; A.F Proofread, revised and finalized the manuscript; All authors read and approved the final manuscript 


\section{Funding}

This work was supported by the Fasa University of Medical sciences

\section{Availability of data and materials}

Not applicable.

\section{Ethics approval and consent to participate}

Not applicable.

\section{Consent for publication}

Not applicable.

\section{Competing interests}

All authors have no competing interests.

\section{References}

1. Diniz E, Tomich de Paula da Silva CH, Gomez-Perez V, Federico LB, Campos Rosa JM. GRIND2-based 3D-QSAR and prediction of activity spectra for symmetrical bis-pyridinium salts with promastigote antileishmanial activity. J Biomol Struct Dyn. 2017;35(11):2430-40

2. Pratti JES, da Fonseca Martins AM, da Silva JP, Ramos TD, Pereira JC, Firmino-Cruz L, et al. The role of TLR9 on Leishmania amazonensis infection and its influence on intranasal LaAg vaccine efficacy. PLoS Negl Trop Dis. 2019;13(2):e0007146.

3. Harley D, latta R, Furlanello T, Colella V, Tarallo VD, Latrofa MS, et al. A nationwide survey of Leishmania infantum infection in cats and associated risk factors in Italy. PLOS Neglected Tropical Diseases. 2019;13(7).

4. Pomel S, Mao W, Ha-Duong T, Cave C, Loiseau PM. GDP-Mannose Pyrophosphorylase: A Biologically Validated Target for Drug Development Against Leishmaniasis. Front Cell Infect Microbiol. 2019;9:186.

5. Gutiérrez V, Seabra AB, Reguera RM, Khandare J, Calderón M. New approaches from nanomedicine for treating leishmaniasis. Chemical Society Reviews. 2016;45(1):152-68.

6. Gholami E, Zahedifard F, Rafati S. Delivery systems for Leishmania vaccine development. Expert Rev Vaccines. 2016;15(7):879-95.

7. Osman M, Mistry A, Keding A, Gabe R, Cook E, Forrester S, et al. A third generation vaccine for human visceral leishmaniasis and post kala azar dermal leishmaniasis: First-in-human trial of ChAd63-KH. PLoS Negl Trop Dis. 2017;11(5):e0005527.

8. Mortazavidehkordi N, Fallah A, Abdollahi A, Kia V, Khanahmad H, Najafabadi ZG, et al. A lentiviral vaccine expressing KMP11-HASPB fusion protein increases immune response to Leishmania major in BALB/C. Parasitol Res. 2018;117(7):2265-73.

9. Avishek K, Kaushal H, Gannavaram S, Dey R, Selvapandiyan A, Ramesh V, et al. Gene deleted live attenuated Leishmania vaccine candidates against visceral leishmaniasis elicit pro-inflammatory cytokines response in human PBMCs. Sci Rep. 2016;6:33059.

10. Fiuza JA, Santiago Hda C, Selvapandiyan A, Gannavaram S, Ricci ND, Bueno LL, et al. Induction of immunogenicity by live attenuated Leishmania donovani centrin deleted parasites in dogs. Vaccine. 2013;31(14):1785-92.

11. Lima MP, Costa LE, Lage DP, Dias DS, Ribeiro PAF, Machado AS, et al. Diagnostic application of recombinant Leishmania proteins and evaluation of their in vitro immunogenicity after stimulation of immune cells collected from tegumentary leishmaniasis patients and healthy individuals. Cell Immunol. 2018;334:61-9.

12. Martins VT, Chavez-Fumagalli MA, Costa LE, Canavaci AM, Martins AM, Lage PS, et al. Antigenicity and protective efficacy of a Leishmania amastigote-specific protein, member of the super-oxygenase family, against visceral leishmaniasis. PLoS Negl Trop Dis. 2013;7(3):e2148.

13. Das A, Ali N. Vaccine Development Against Leishmania donovani. Front Immunol. 2012;3:99.

14. SUN L, JLXX. Overcoming adeno-associated virus vector size limitation through viral DNA heterodimerization. NEW TECHNOLOGY. 2000.

15. Khan M, Khan S, Ali A, Akbar H, Sayaf AM, Khan A, et al. Immunoinformatics approaches to explore Helicobacter Pylori proteome (Virulence Factors) to design B and T cell multi-epitope subunit vaccine. Sci Rep. 2019;9(1):13321. 
16. Purcell AW, McCluskey J, Rossjohn J. More than one reason to rethink the use of peptides in vaccine design. Nature Reviews Drug Discovery. 2007;6(5):404-14.

17. Khan MA, Hossain MU, Rakib-Uz-Zaman SM, Morshed MN. Epitope-based peptide vaccine design and target site depiction against Ebola viruses: an immunoinformatics study. Scand J Immunol. 2015;82(1):25-34.

18. Fleri W, Paul S, Dhanda SK, Mahajan S, Xu X, Peters B, et al. The Immune Epitope Database and Analysis Resource in Epitope Discovery and Synthetic Vaccine Design. Front Immunol. 2017;8:278.

19. Validi M, Karkhah A, Prajapati VK, Nouri HR. Immuno-informatics based approaches to design a novel multi epitope-based vaccine for immune response reinforcement against Leptospirosis. Mol Immunol. 2018;104:128-38.

20. Ponomarenko J, Bui HH, Li W, Fusseder N, Bourne PE, Sette A, et al. ElliPro: a new structure-based tool for the prediction of antibody epitopes. BMC Bioinformatics. 2008;9:514.

21. Zhang Y. I-TASSER server for protein 3D structure prediction. BMC Bioinformatics. 2008;9:40.

22. Ko J, Park H, Heo L, Seok C. GalaxyWEB server for protein structure prediction and refinement. Nucleic Acids Res. 2012;40(Web Server issue):W294-7.

23. Laskowski RA, et al. PROCHECK: a program to check the stereochemicai quality of protein structure. Applied Crystallography. 1993.

24. Wiederstein M, Sippl MJ. ProSA-web: interactive web service for the recognition of errors in three-dimensional structures of proteins. Nucleic Acids Res. 2007;35(Web Server issue):W407-10.

25. Gasteiger Eea. Protein identification and analysis tools on the ExPASy server. Springer. 2005.

26. Magnan CN, Randall A, Baldi P. SOLpro: accurate sequence-based prediction of protein solubility. Bioinformatics. 2009;25(17):2200-7.

27. Dimitrov I, Naneva L, Doytchinova I, Bangov I. AllergenFP: allergenicity prediction by descriptor fingerprints. Bioinformatics. 2014;30(6):846-51.

28. Dimitrov I, Bangov I, Flower DR, Doytchinova I. AllerTOP v.2--a server for in silico prediction of allergens. J Mol Model. 2014;20(6):2278.

29. Magnan CN, Zeller M, Kayala MA, Vigil A, Randall A, Felgner PL, et al. High-throughput prediction of protein antigenicity using protein microarray data. Bioinformatics. 2010;26(23):2936-43.

30. Doytchinova IA, Flower DR. VaxiJen: a server for prediction of protective antigens, tumour antigens and subunit vaccines. BMC Bioinformatics. 2007;8:4.

31. Kozakov D, Hall DR, Xia B, Porter KA, Padhorny D, Yueh C, et al. The ClusPro web server for protein-protein docking. Nat Protoc. 2017;12(2):255-78.

32. Jardim A, Davenport BJ, Martin CG, Beverley SM, Orlicky DJ, Vazquez-Torres A, et al. SODB1 is essential for Leishmania major infection of macrophages and pathogenesis in mice. PLOS Neglected Tropical Diseases. 2018;12(10).

33. Sherif El Shanat MB. Leishmaniasis: Epidemiology, Control and Future Perspectives with Special Emphasis on Egypt. Journal of Tropical Diseases. 2015;03(01).

34. Petitdidier E, Pagniez J, Pissarra J, Holzmuller P, Papierok G, Vincendeau P, et al. Peptide-based vaccine successfully induces protective immunity against canine visceral leishmaniasis. npj Vaccines. 2019;4(1).

35. Khan M, Khan S, Ali A, Akbar H, Sayaf AM, Khan A, et al. Immunoinformatics approaches to explore Helicobacter Pylori proteome (Virulence Factors) to design B and T cell multi-epitope subunit vaccine. Scientific Reports. 2019;9(1).

36. Ahmed SBH, Bahloul C, Robbana C, Askri S, Dellagi K. A comparative evaluation of different DNA vaccine candidates against experimental murine leishmaniasis due to L. major. Vaccine. 2004;22(13-14):1631-9.

37. Yarmarkovich M, Warrington JM, Farrel A, Maris JM. Identification of SARS-CoV-2 Vaccine Epitopes Predicted to Induce Long-Term Population-Scale Immunity. Cell Reports Medicine. 2020;1(3).

38. Hedhli D, Moiré N, Akbar H, Laurent F, Héraut B, Dimier-Poisson I, et al. The antigen-specific response to Toxoplasma gondii profilin, a TLR11/12 ligand, depends on its intrinsic adjuvant properties. Medical Microbiology and Immunology. 2016;205(4):345-52.

\section{Figures}




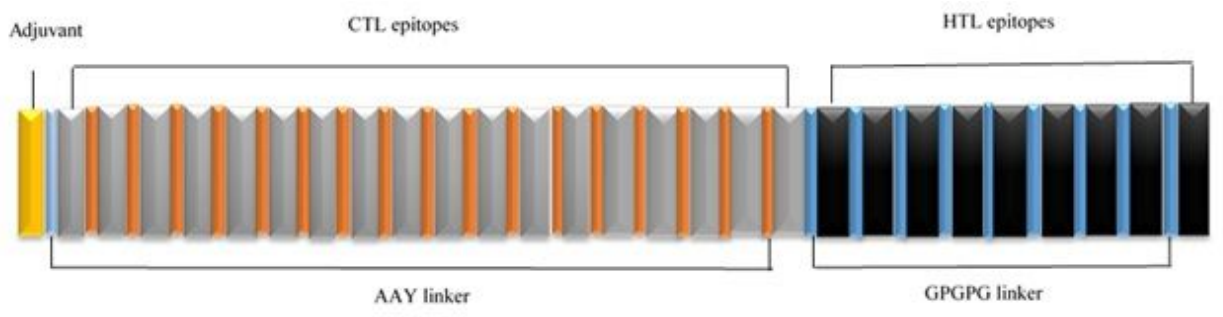

\section{Figure 1}

Schematic diagram of final multi-epitope vaccine. The construct consists of 18 CTL epitopes with gray color that joined by AAY linker. There are also 9 HTL epitopes in the structure of the final vaccine, which are shown in black color and were joined by GPGPG linker. The profilin adjuvant was also added into the construct by AAY Linker. There are 613 residues in the structure of the final construct.
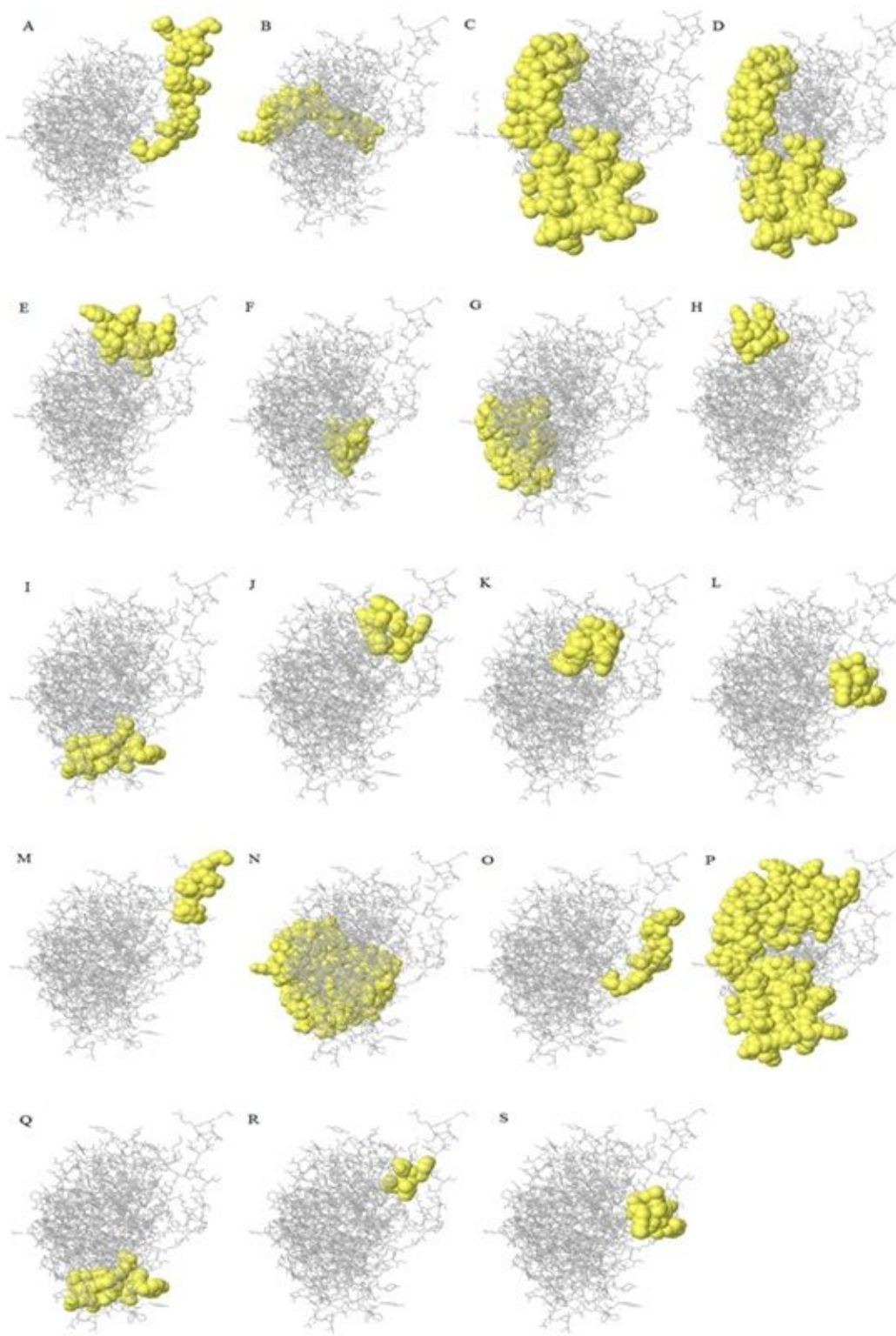

\section{Figure 2}

B cell epitope prediction. (A-L) indicate linear epitopes and M-S are conformational epitopes. 
A

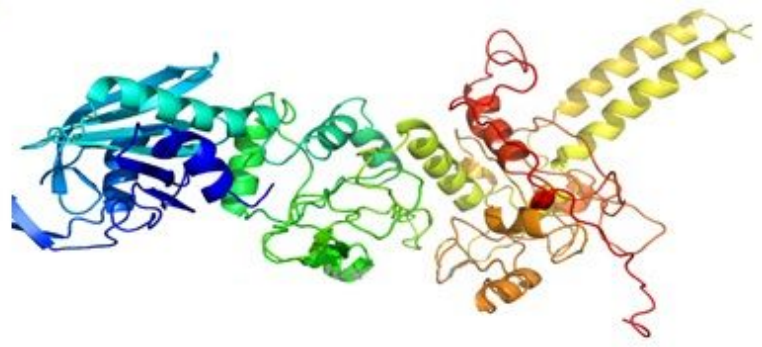

B

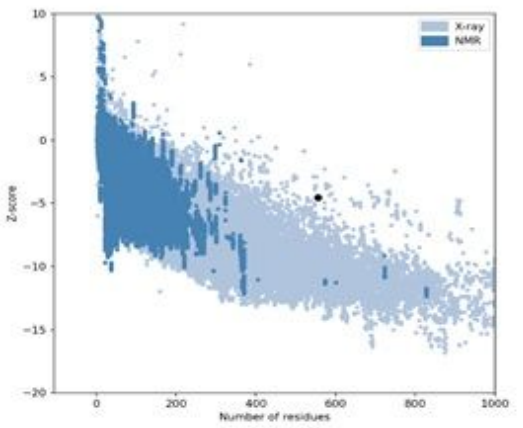

C

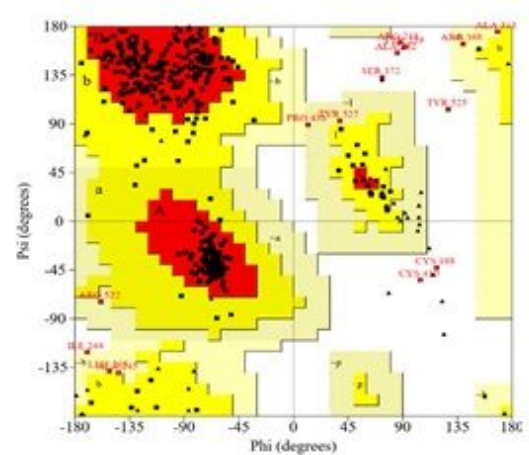

Figure 3

Three dimensional structure of the final vaccine construct. (A) Prediction of 3D structure of final multi-epitope vaccine that was obtained by using I-TASSER server. (B) Validation of 3D predicted model by ProSA gave a Z-score with a value of -4.55 (C) Ramachandran plot indicated $87.0 \%$ of residues existed in favored regions, $9.9 \%$ in additional regions, $1.5 \%$ in generously allowed and $1.5 \%$ in and disallowed regions, respectively.

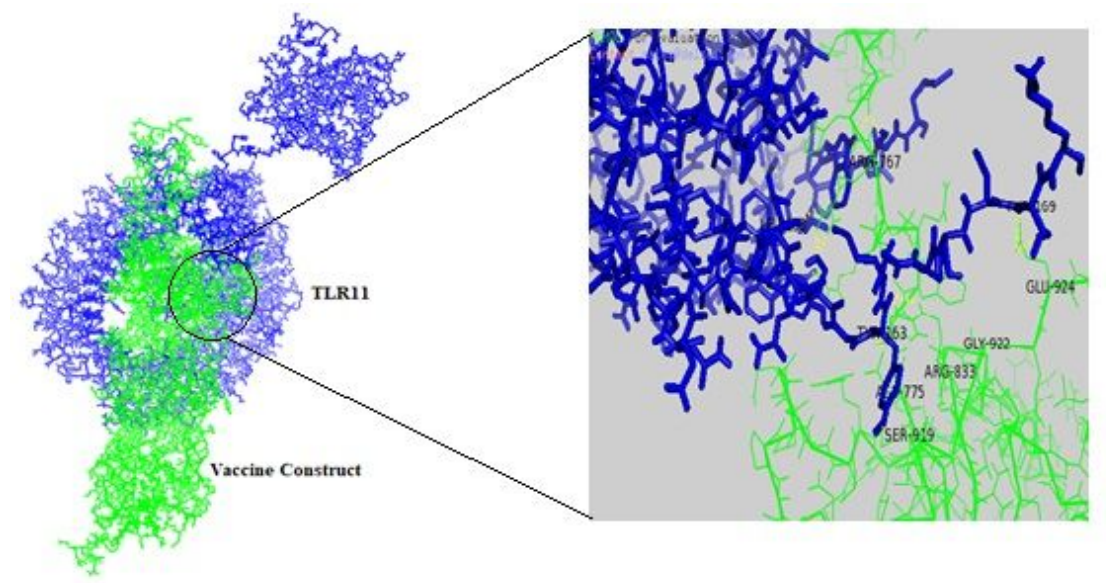

\section{Figure 4}

Docking analysis of final multi-epitope vaccine and TLR11. The blue color indicates receptor (TLR11) and the green color indicates the ligand (final multi-epitope vaccine). 
A

RMSD

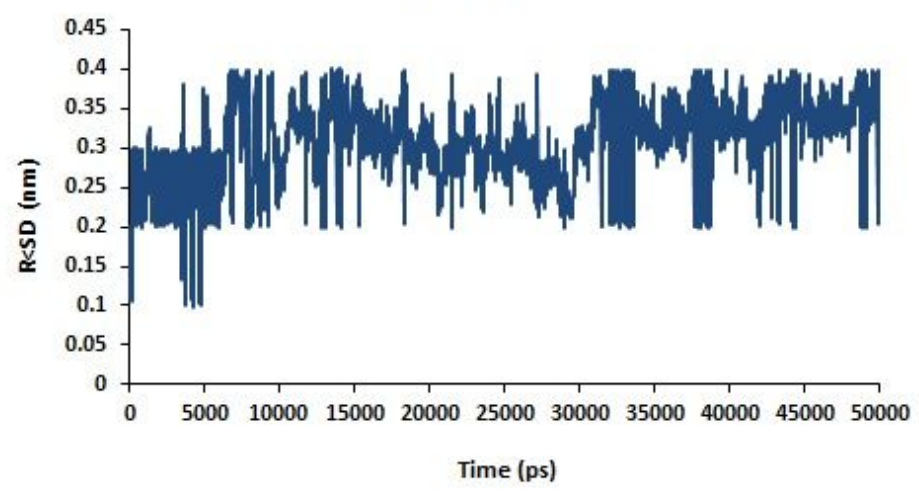

B

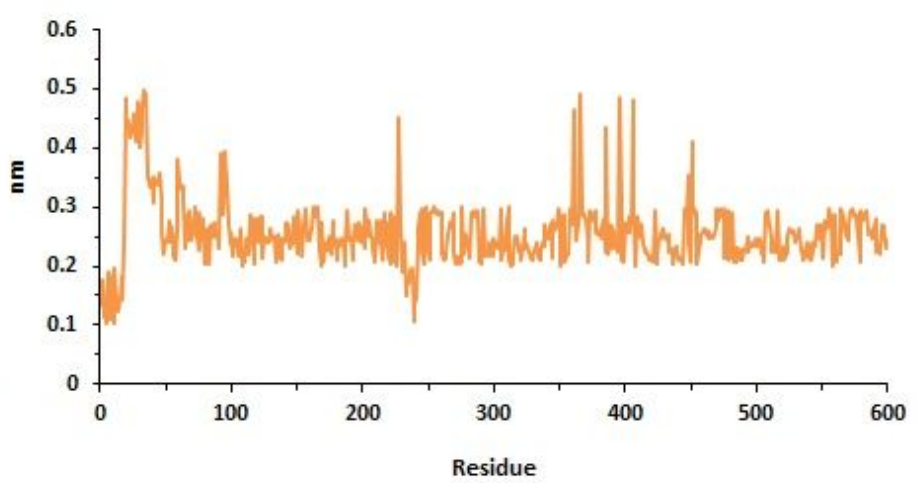

\section{Figure 5}

Molecular dynamics simulation of final vaccine and TLR-11. (A) The RMSD plot of final vaccine-TLR11 during 30000 Ps. (B) The RMSF plot of final vaccine at the same time.

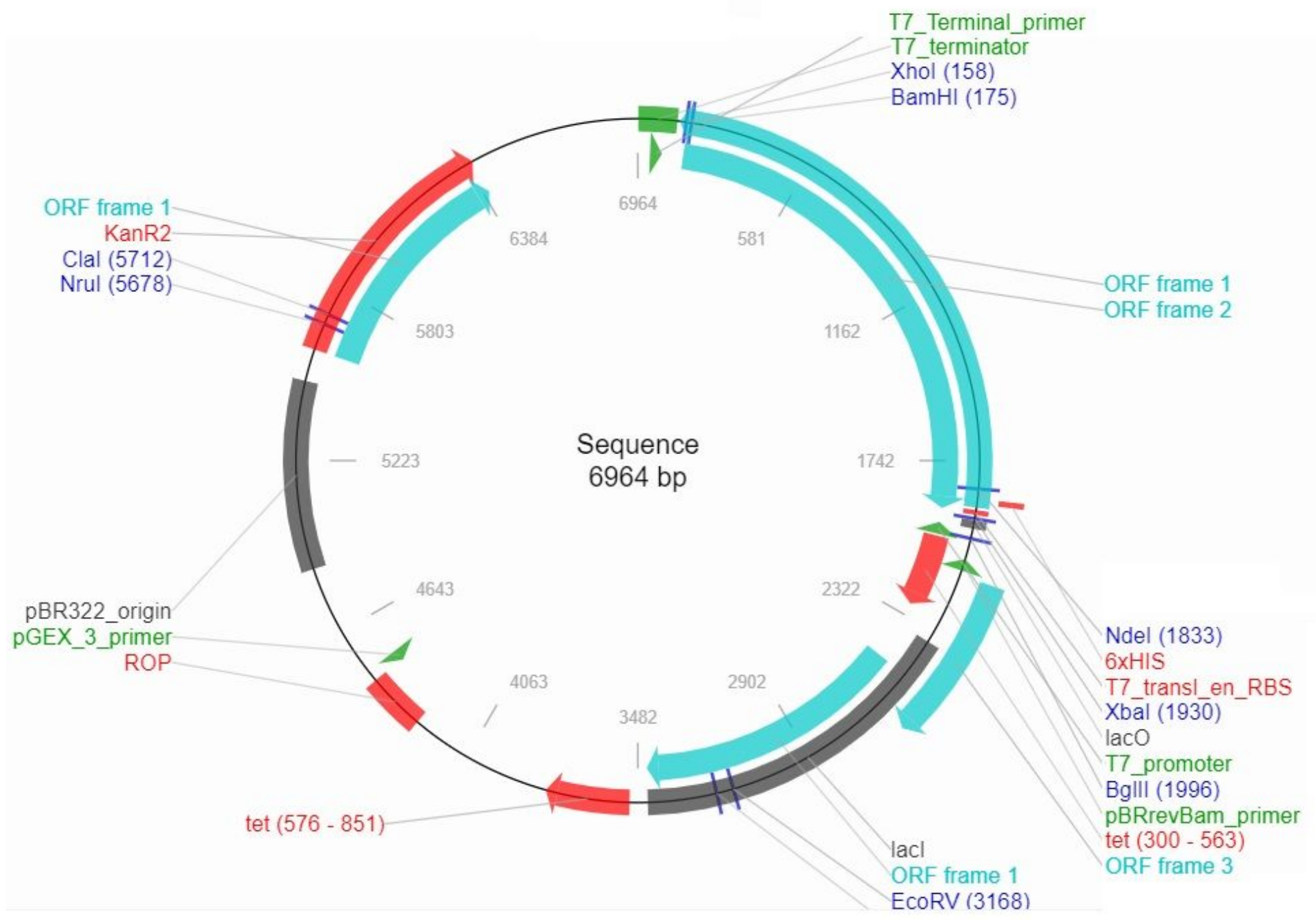

\section{Figure 6}

In silico cloning of final multi-epitope vaccine. The purple line indicates where the vaccine is attached to the vector and the black line indicates the vector. 